\title{
Dendritic cell-based immunotherapy targeting Wilms' tumor 1 in patients with recurrent malignant glioma
}

\author{
Keiichi Sakai, MD, PhD, ${ }^{1,11}$ Shigetaka Shimodaira, MD, PhD, ${ }^{2}$ Shinya Maejima, MD, PhD, ${ }^{3}$ \\ Nobuyuki Udagawa, DDS, PhD, ${ }^{3}$ Kenji Sano, MD, PhD, ${ }^{4}$ Yumiko Higuchi, PhD, ${ }^{2}$ \\ Terutsugu Koya, MS, ${ }^{2}$ Takanaga Ochiai, DDS, PhD, ${ }^{5}$ Masanori Koide, DDS, PhD, ${ }^{6}$ \\ Shunsuke Uehara, $\mathrm{PhD},{ }^{7}$ Midori Nakamura, DDS, PhD, ${ }^{7}$ Haruo Sugiyama, MD, $\mathrm{PhD},{ }^{8}$ \\ Yoshikazu Yonemitsu, MD, PhD, ${ }^{9}$ Masato Okamoto, DDS, PhD, ${ }^{10}$ and Kazuhiro Hongo, MD, PhD ${ }^{1}$
}

1Department of Neurosurgery, Shinshu University School of Medicine; ${ }^{2}$ Center for Advanced Cellular Therapy, Shinshu University Hospital, Matsumoto; ${ }^{3}$ Dendritic Cell Vaccination Therapy Center, ${ }^{5}$ Department of Oral Pathology, ${ }^{6}$ Institute for Oral Science, and 'Department of Biochemistry, Matsumoto Dental University Hospital, Shiojiri; ${ }^{4}$ Department of Laboratory, Shinshu University Hospital, Matsumoto; ${ }^{8}$ Department of Functional Diagnostic Science, Graduate School of Medicine, Osaka University, Osaka; ${ }^{2}$ R\&D Laboratory for Innovative Biotherapeutics, Graduate School of Pharmaceutical Sciences, Kyushu University, Higashi-ku, Fukuoka; ${ }^{10}$ Department of Advanced Immunotherapeutics, Kitasato University School of Pharmacy, Minato-ku, Tokyo; and ${ }^{11}$ Department of Neurosurgery, National Hospital Organization, Shinshu Ueda Medical Center, Ueda, Nagano, Japan

OBJECT Dendritic cell (DC)-based vaccination is considered a potentially effective therapy against advanced cancer. The authors conducted a Phase I study to investigate the safety and immunomonitoring of Wilms' tumor 1 (WT1)-pulsed DC vaccination therapy for patients with relapsed malignant glioma.

METHODS WT1-pulsed and/or autologous tumor lysate-pulsed DC vaccination therapy was performed in patients with relapsed malignant gliomas. Approximately $1 \times 10^{7}$ to $2 \times 10^{7}$ pulsed DCs loaded with WT1 peptide antigen and/or tumor lysate were intradermally injected into the axillary areas with OK-432, a streptococcal preparation, at 2-week intervals for at least $5-7$ sessions (1 course) during an individual chemotherapy regimen.

RESULTS Ten patients ( 3 men, 7 women; age range 24-64 years [median 39 years]) with the following tumors were enrolled: glioblastoma (6), anaplastic astrocytoma (2), anaplastic oligoastrocytoma (1), and anaplastic oligodendroglioma (1). Modified WT1 peptide-pulsed DC vaccine was administered to 7 patients, tumor lysate-pulsed DC vaccine to 2 patients, and both tumor lysate-pulsed and WT1-pulsed DC vaccine to 1 patient. The clinical response was stable disease in 5 patients with WT1-pulsed DC vaccination. In 2 of 5 patients with stable disease, neurological findings improved, and MR images showed tumor shrinkage. No serious adverse events occurred except Grade 1-2 erythema at the injection sites. WT1 tetramer analysis detected WT1-reactive cytotoxic T cells after vaccination in patients treated with WT1pulsed therapy. Positivity for skin reaction at the injection sites was $80 \%$ ( 8 of 10 patients) after the first session, and positivity remained for these 8 patients after the final session.

CONCLUSIONS This study of WT1-pulsed DC vaccination therapy demonstrated safety, immunogenicity, and feasibility in the management of relapsed malignant gliomas.

http://thejns.org/doi/abs/10.3171/2015.1.JNS141554

KEY WORDS relapsed malignant glioma; dendritic cell; WT1; immunotherapy; oncology

ABBREVIATIONS CTL = cytotoxic $T$ lymphocyte; $D C=$ dendritic cell; $H L A=$ human leukocyte antigen; IL = interleukin; Ke = kiloequivalent; KPS = Karnofsky Performance Scale; MST = median overall survival time; $\mathrm{PD}$ = progressive disease; $\mathrm{PR}=$ partial response; $\mathrm{RECIST}=$ Response Evaluation Criteria in Solid Tumors; $\mathrm{SD}=$ stable disease; TAA = tumor-associated antigen; WT1 = Wilms' tumor 1 .

SUBMITTED July 13, 2014. ACCEPTED January 20, 2015.

INCLUDE WHEN CITING Published online August 7, 2015; DOI: 10.3171/2015.1.JNS141554.

DISCLOSURE Prof. Yonemitsu is a previous member of the Board of Directors on Science and Medicine at tella, Inc.; Prof. Okamoto is a stockholder of tella, Inc.; and Prof. Sugiyama holds ownership interest in Dainippon Sumitomo Pharmaceutical Co., including patents for the WT1 peptide. These 3 authors did not participate in the data analyses. 
$\mathrm{G}$ LIOMAS are the most common type of brain tumor. Patients with high-grade gliomas, including those with glioblastomas, have a poor prognosis. $.^{38,40} \mathrm{De}-$ spite recent multimodal treatment including surgery, radiation therapy, and chemotherapy, no significant improvement has been reported in overall survival. ${ }^{1}$ In patients with newly diagnosed glioblastoma, the median survival time is 14.6 months, ${ }^{40}$ and the 5-year survival rate is $9 \% .{ }^{39}$ The prognosis for those with relapsed malignant glioma is extremely poor. Therefore, a strong need exists for novel therapeutic strategies against malignant gliomas.

Immunotherapy is a new promising therapeutic approach that can specifically target tumor cells. ${ }^{46}$ Most immunotherapeutic strategies are focused on inducing specific immune responses against tumor-associated antigens (TAAs). Several clinical trials using vaccination with synthetic peptides of TAAs have been performed. TAAs associated with glioblastomas have been described and include epidermal growth factor receptor vIII, interleukin-13R $\alpha 2$ (IL-13R $\alpha 2$ ), IL-4, Wilms' tumor 1 (WT1), survivin, and erythropoietin-producing hepatocellular receptor tyrosine kinase class A2 (EphA2). ${ }^{13,33,35}$ These studies, however, have shown limited clinical efficacy.

WT1 is one of a large number of TAAs and is thought to function as an oncogene. ${ }^{26,42}$ WT1 was isolated as the causative gene of Wilms' tumor, which is a childhood renal tumor. The WT1 gene encodes a transcription factor that controls the transcription of various genes and is overexpressed in a variety of hematological malignancies and solid tumors, including gliomas. ${ }^{12,27} \mathrm{WT} 1$ may be a target antigen for immunotherapy against these cancers. ${ }^{29}$ Phase I and II clinical trials have been conducted with the WT1 peptide to treat various cancers, and the safety and efficacy results have been promising. ${ }^{10,17,21,29}$ The WT1 antigen was designated "a cancer antigen having highest priority" in "The Prioritization of Cancer Antigens: A National Cancer Institute Pilot Project for the Acceleration of Translational Research."6

Vaccination with dendritic cells (DCs) pulsed with TAAs is another recent promising immunotherapeutic approach for patients with carcinoma. ${ }^{18,43}$ DCs are hematopoietically derived cells that act as antigen-presenting cells to activate innate and adaptive immune responses. For this approach, DCs are extracted from the patient and exposed to antigens expressed by the tumor type to be treated. Recent Phase I and II studies demonstrated the safety and efficacy of DC vaccination against malignant gliomas. ${ }^{5,16,36}$ Vaccination with DCs was also studied in 313 patients with high-grade gliomas. ${ }^{44}$ The antigen sources were tumor lysate, peptides eluted from autologous tumor cells, defined peptides, and autologous tumor cells. Phase I and II data suggest that DC vaccination potentially offers a new approach in the treatment of malignant gliomas.

We previously conducted DC vaccination therapy in patients with advanced cancer and reported the safety and efficacy of these treatments. ${ }^{18,19,43}$ This new DC-based vaccination approach may be an effective therapy against advanced cancer. Our protocol using mature DCs pulsed with WT1 peptide and/or using induced mature DCs from immature DCs pulsed with autologous tumor lysate against malignant gliomas has not been reported previously. Here, we investigated the safety and immunogenicity of WT1pulsed DC vaccination therapy in patients with malignant gliomas that had relapsed after the standard treatment.

\section{Methods}

\section{Patients}

This clinical trial was approved by the Ethical Committee of Shinshu University School of Medicine (December 2, 2008, approval number 1199) and the Ethical Committee of Matsumoto Dental University (February 25, 2010, approval number 0102). Written informed consent was obtained from each patient on a document approved by the committee of the applicable institution. Each patient had a relapsed malignant glioma. The inclusion criteria were recurrence of a malignant glioma after the standard treatment and chemotherapy- and/or radiotherapy-resistant progressive disease (PD).

\section{Vaccination}

WT1 Class I peptides for human leukocyte antigens (HLAs) HLA-A*24:02 and HLA-A*02:01 were preferentially chosen for patients harboring HLA-A*24:02 and HLA-A*02:01/A*02:06 typing, respectively. We established the standard of WT1 expression using immunohistochemistry of cell lines. First, mRNA expression of cell lines including WT1-positive (Panc 1 and Mia-Paca2) and WT1-negative (HCT116 and MCF7) cell lines was confirmed with real-time polymerase chain reaction. WT1 immunohistochemistry of those cell lines revealed expression that was proportional to the mRNA level. In reference to those cell lines, WT1 expression was evaluated with immunohistochemical staining on paraffin-embedded tumor tissues obtained during surgery. ${ }^{25}$ Anti-WT1 is a mouse monoclonal antibody (6F-H2, DakoCytomation). If WT1 expression was observed with immunohistochemistry, the cell line was considered positive; if no expression was observed, it was considered negative. If frozen tumor tissues were stored, a tumor lysate was prepared for loading DCs as previously described. ${ }^{20,24}$ DCs were prepared as previously described. . $^{9} 18,31,34$

According to the vaccination protocol, the DCs were cryopreserved until the day of administration. An aliquot of frozen mature DCs was thawed and loaded with 100 ug/ml HLA-A*24:02-restricted mutant WT1 peptide (residues 235-243: CYTWNQMNL) or $\mathrm{A}^{* 02: 01 / \mathrm{A}^{*} 02: 06-}$ restricted wild-type WT1 peptide (residues 126-134: RMFPNAPYL), both of which were GMP (good manufacturing practice) grade and derived from NeoMPS just before clinical use. Approximately $1 \times 10^{7}$ to $2 \times 10^{7} \mathrm{DCs}$ were injected intradermally into the axillary areas with OK-432 (1-5 kiloequivalents [Ke]/dose), a streptococcal preparation, at 2-week intervals for at least 5 to 7 sessions (1 course) during an individual chemotherapy regimen. ${ }^{28,29}$ If a positive response to treatment or no adverse effect (as described below in Clinical Assessments) was observed after 1 course of vaccination, additional vaccinations were given with the patient's informed consent.

\section{Skin Reactions}

The skin's reaction at the injection site was examined 
after the first and seventh vaccinations. Redness and induration were assessed after 24, 48, and 72 hours. Any injection site reaction was measured as the maximum diameter of erythema and induration. A positive skin reaction was defined as erythema and induration of more than $3 \mathrm{~mm}$.

\section{Cell Surface Analysis (Flow Cytometry)}

Peripheral blood mononuclear cells were obtained after the first and seventh vaccinations. The phenotypes of circulating T-cell populations were determined with fluorescence-activated cell sorting by measuring the total $\mathrm{CD}^{+}$ population, the $\mathrm{CD}^{+}{ }^{+}$subpopulation, the $\mathrm{CD}^{+}$subpopulation, the activation markers HLA-DR on CD3 ${ }^{+}$cells, CD19 and $\mathrm{CD} 56$ on $\mathrm{CD}^{+}$cells, and $\mathrm{CD}^{2} 5^{+}$on $\mathrm{CD}^{+}$cells, and the WT1 tetramer on $\mathrm{CD}^{+}$cells.

\section{WT1 Tetramer Analysis}

Levels of WT1-specific cytotoxic $\mathrm{T}$ lymphocytes (CTLs) were evaluated with tetramer analysis (Medical \& Biological Laboratories Co., Ltd.) before and after the course of vaccination. ${ }^{34}$

\section{Clinical Assessments}

Patients were followed up with clinical examination and MRI every 2-3 months. The Karnofsky Performance Scale (KPS) was assessed at each visit. Tumor regression was assessed by using MRI and evaluated according to the Response Evaluation Criteria in Solid Tumors (RECIST) guidelines. Adverse effects were monitored and graded according to the National Cancer Institute Common Toxicity Criteria version 4.0.

\section{Results \\ Patient Characteristics}

Between June 2010 and November 2012, we treated 10 patients ( 3 men, 7 women; median age 39 years [range 2464 years]), including 6 with glioblastoma, 2 with anaplastic astrocytoma, 1 with anaplastic oligoastrocytoma, and 1 with anaplastic oligodendroglioma (Table 1). Before vaccination, each patient had undergone surgery and conventional radiotherapy and had been treated with 1 or more courses of chemotherapy. After relapse, tumor resection was performed in 4 patients, and stereotactic radiotherapy was performed in 3 patients. All except one patient received second- or third-line chemotherapy during vaccination therapy. All except one patient (Patient 3) were given oral steroids before vaccination. Before vaccination, the median KPS score was $80 \%$ (range 40\%-90\%).

\section{Vaccine Preparation and Characterization}

Details of the immunological outcome of each patient after the vaccinations are listed in Tables 1 and 2. A flow diagram for the 10 patients is shown in Fig. 1. Seven patients received WT1 peptide-pulsed vaccination restricted to HLA-A*24:02. One patient received both tumor lysate-pulsed and WT1-A*24:02-pulsed vaccination. Two patients received tumor lysate-pulsed vaccination without WT1, because immunostaining for WT1 was negative. The immature DCs were stimulated with OK-432, a streptococcal preparation $(10 \mu \mathrm{g} / \mathrm{ml})$, and prostaglandin E2 (50 ng/ml; Daiichi Fine Chemical Co., Ltd.), IL-4 (5 $\mathrm{ng} / \mathrm{ml})$, and granulocyte-macrophage colony-stimulating factor $(5 \mathrm{ng} / \mathrm{ml})$ for 24 hours to produce mature DCs. ${ }^{18,19}$ The final yield of mature DCs was $0.37 \times 10^{7}$ to $32.75 \times$ $10^{7}$ cells (median $9.9 \times 10^{7}$ cells). However, Patient 4 , in whom the final yield of DCs was less than $4.51 \times 10^{7}$, had received oral steroid therapy for a long time before the onset of her brain tumor for a skin disease. A median of $0.97 \times 10^{7}$ (range $0.07 \times 10^{7}$ to $9.94 \times 10^{7} \mathrm{DCs}$ ) mature DCs were injected per vaccine lot. A median of 7 vaccinations (range 5-7 vaccinations) were given as the first course. Seven patients received more than 11 vaccinations (range 11-23 vaccinations). The total dose of OK-432 was 19-89 $\mathrm{Ke}$ (median $24 \mathrm{Ke}$ ). The maximum dose of OK-432 per session was $5 \mathrm{Ke}$. The phenotypes of the DCs were determined by the expression of CD11c, CD40, CD80, CD83, CD86, and HLA-DR.

\section{Immune Response}

A skin reaction was observed in 8 patients after the first and final vaccinations of the first course; in Patients 4 and 10 , a skin reaction was observed only after the first vaccination. The skin reaction remained positive in all 8 patients after the final session. In 1 patient, the skin reaction after the first vaccination was negative but became positive after the seventh session. No correlation was found between skin reaction and progression-free survival or overall survival.

A tetramer assay was performed for each of the 8 patients treated with WT1-pulsed therapy. The WT1/CTL ratio was elevated after vaccination in 6 patients (Table 2 ). In those 6 patients, a $>2$-fold increase in the WT1/ CTL ratio was noted. In Patient 4 , no increase in the WT1/ CTL ratio was observed. The patient had received longterm oral steroids for a skin disease before the onset of her brain tumor. Two of the 6 patients with an increased WT1/ CTL ratio had a partial response (PR) according to the RECIST during the clinical course. No correlation was found between the results of the tetramer assay and positive skin test results.

\section{Clinical Outcome}

Response characteristics and applied treatments after the first DC vaccination in each patient are shown in Fig. 2. At the end of the first session of vaccinations, 5 patients showed stable disease (SD), and 5 patients showed PD. Each patient who had SD had been treated with WT1pulsed DC therapy. One of the 6 patients with glioblastoma showed SD, but he died 6 months after the first session. In 2 of the 5 patients with SD, the KPS score improved from $40 \%$ to $50 \%$ (Patient 1 ) and from $70 \%$ to $80 \%(\mathrm{~Pa}-$ tient 2). MRI of Patients 1 and 2 revealed tumor shrinkage after vaccination. A PR was noted during the continuous vaccination after the first session. Neurological findings improved in 3 patients (Patients 3-5), although their KPS scores did not improve.

With a maximum follow-up time of 21 months after the first session of DC vaccination, the final outcome was death in 5 patients. The survival time from the initial diag- 


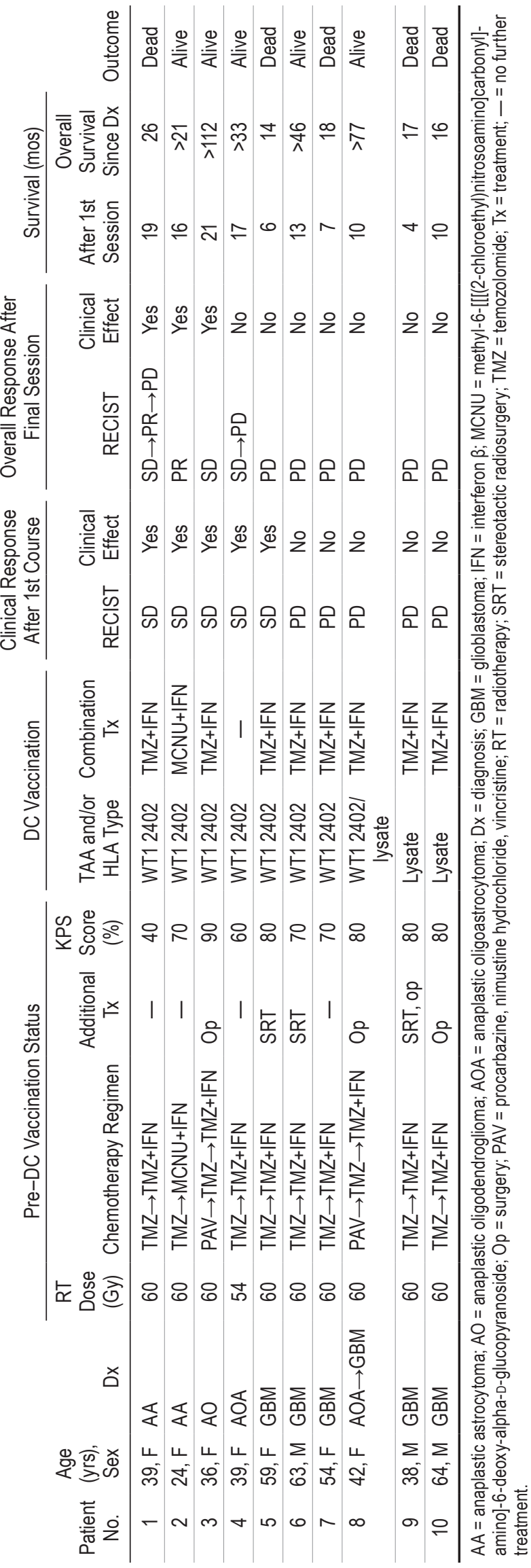

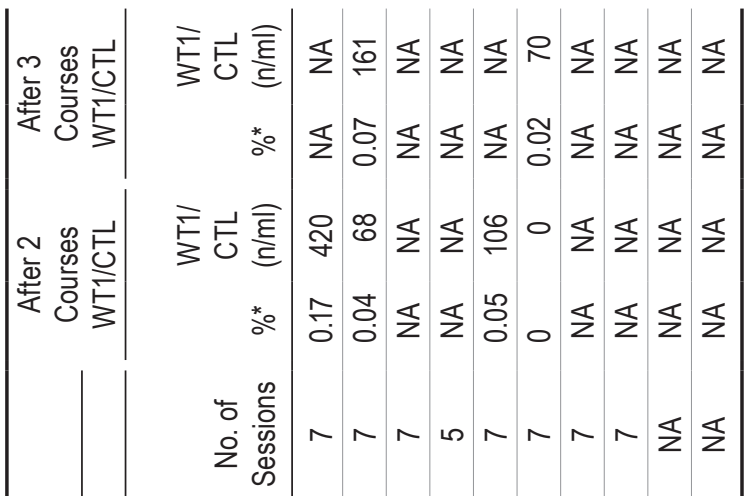

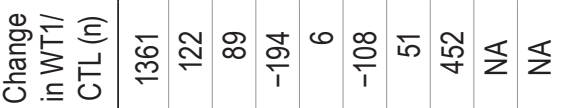

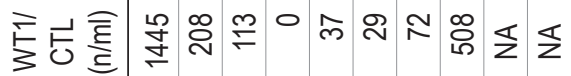

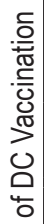
兽는

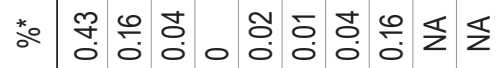
产部 일

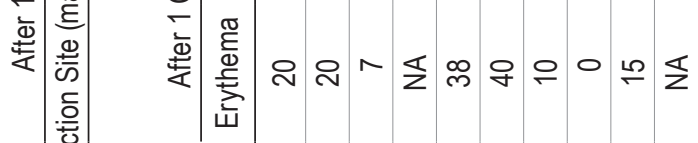

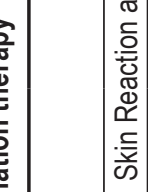

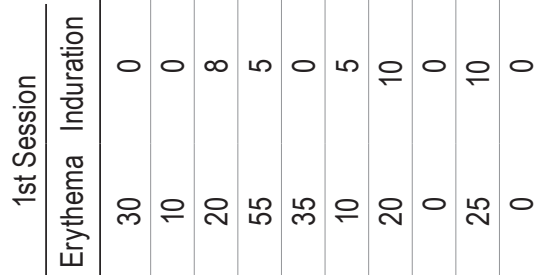

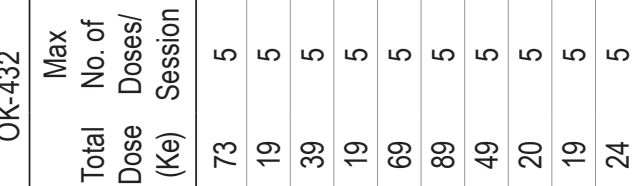

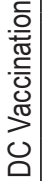

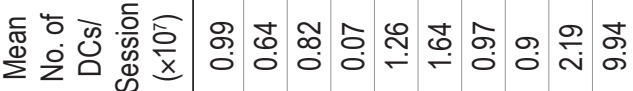

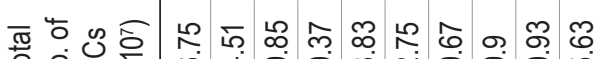

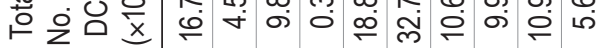

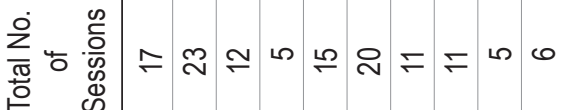

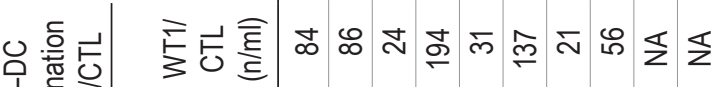

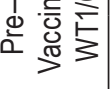

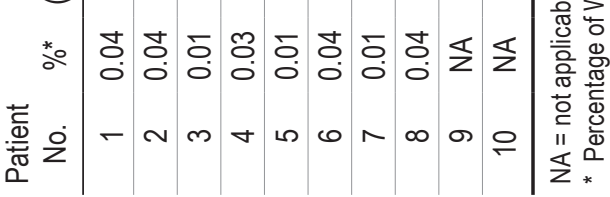




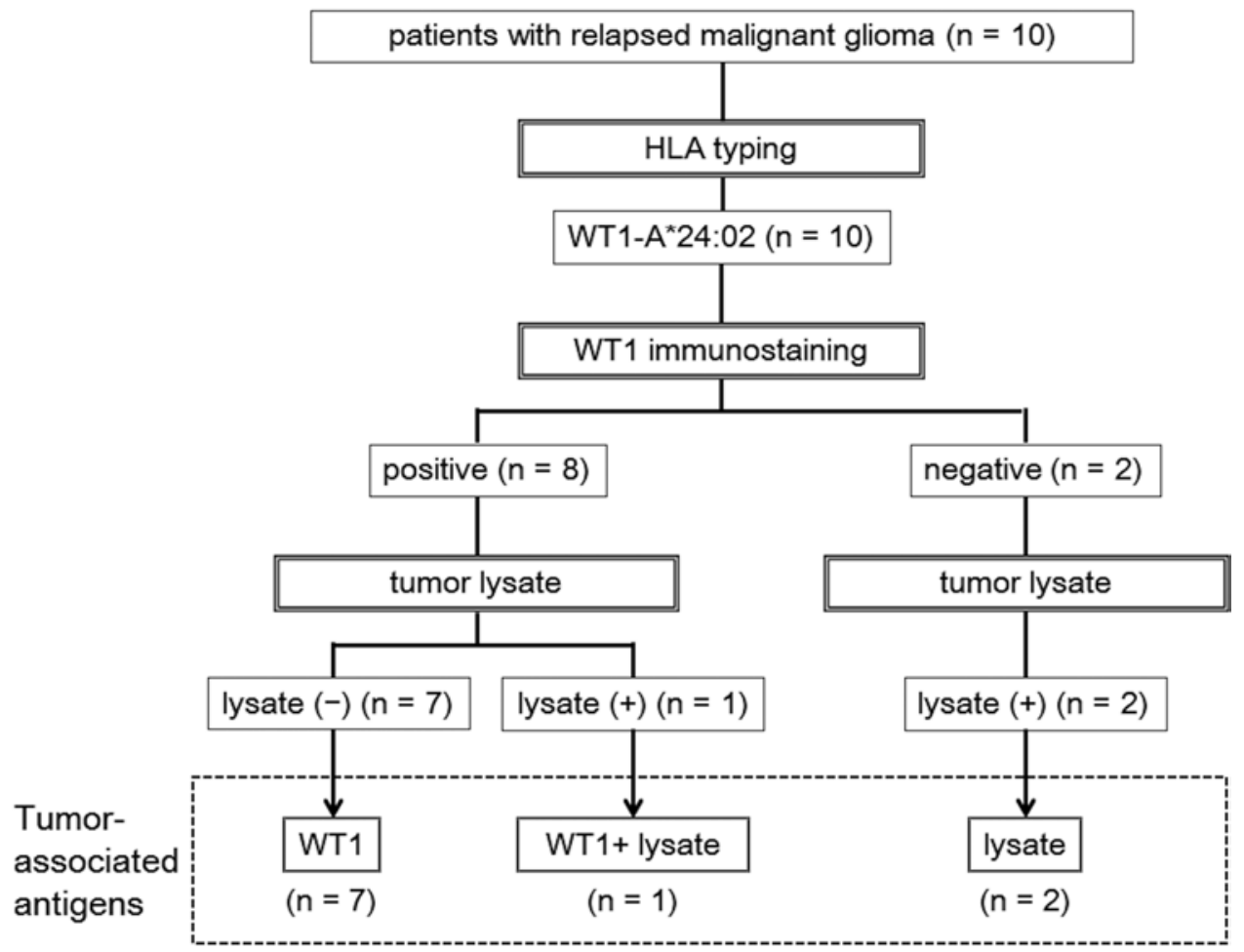

FIG. 1. Flow diagram of the 10 patients with recurrent malignant gliomas.

nosis was $14-112$ months, and that from the first DC vaccination was 12.3 months (range 4-21 months). As shown in Fig. 3, the median overall survival time (MST) of all patients after the initial diagnosis was 26 months, and the MST of the patients with glioblastoma was 18 months (range 14-77 months). The MST of all patients after the first vaccination was 19 months, and the MST of the patients with glioblastoma was 7 months. Of the 5 patients with primary glioblastoma, 5 had PD, and 4 of them died. However, 1 patient with glioblastoma (Patient 6) survived 13 months after the first session of vaccination and was still alive 46 months after the initial diagnosis.

\section{Safety and Toxicity}

No Grade 3 or higher National Cancer Institute Common Toxicity Criteria toxicities associated with vaccination were found. Mild adverse events, including mild erythema at the injection site that was observed in every patient within a few days, were noted. Postvaccination fever was seen in 6 patients $(60 \%)$, and fatigue was noted in 3 patients (30\%).

\section{Discussion}

We report here the results of a pilot study of DC-based immunotherapy targeting WT1 for patients with relapsed malignant glioma. SD was attained after the first course in $5(62.5 \%)$ of the 8 patients who received WT1-pulsed therapy. A PR was achieved in $25 \%$ of the patients (Patients 1 and 2) during continuous vaccination after the first session. In 2 patients with favorable clinical and radiological responses, the WT1/CTL ratio was predominantly increased. In all patients except one, an increase in the ratio of peripheral WT1/CTL after WT1-pulsed DC vaccination was observed. The toxicities observed in this study were all of Grade 1 or 2 of the National Cancer Institute Common Toxicity Criteria. WT1-pulsed and/or autologous tumor lysate-pulsed DC-based vaccination may be safe and well tolerated in and feasible for the treatment of patients with relapsed malignant gliomas.

Several clinical trials using DC vaccination to treat patients with glioblastoma have been reported, and they demonstrated 1) a trend toward prolonged progression-free survival and overall survival, 2) an association between enhanced cytokine responses and vaccine responders and a correlation with survival, and 3) that DC vaccination is well tolerated and safe. , $^{2,8,85}$ DC-based vaccination has several advantages for the induction and activation of tumor antigen-specific CTLs over vaccination with CTL epitopes alone. ${ }^{22}$ The frequency of WT1-specific CTLs does not increase after WT1 peptide vaccination therapy in patients with recurrent glioblastoma. ${ }^{15}$ In our study, WT1-specific immune responses were markedly increased according to WT1 tetramer analysis after DC vaccination. The frequency and number of WT1-specific CTLs detected by WT1 HLA-A*2402 tetramers were increased after the DC vaccination in most patients. In 6 of 8 patients, a $>2$-fold increase in the WT1/CTL ratio after WT1-pulsed DC vaccination was noted. Scheibenbogen et al. ${ }^{37}$ reported that spontaneous WT1-specific T-cell responses were observed in patients with acute myeloid leukemia. The 


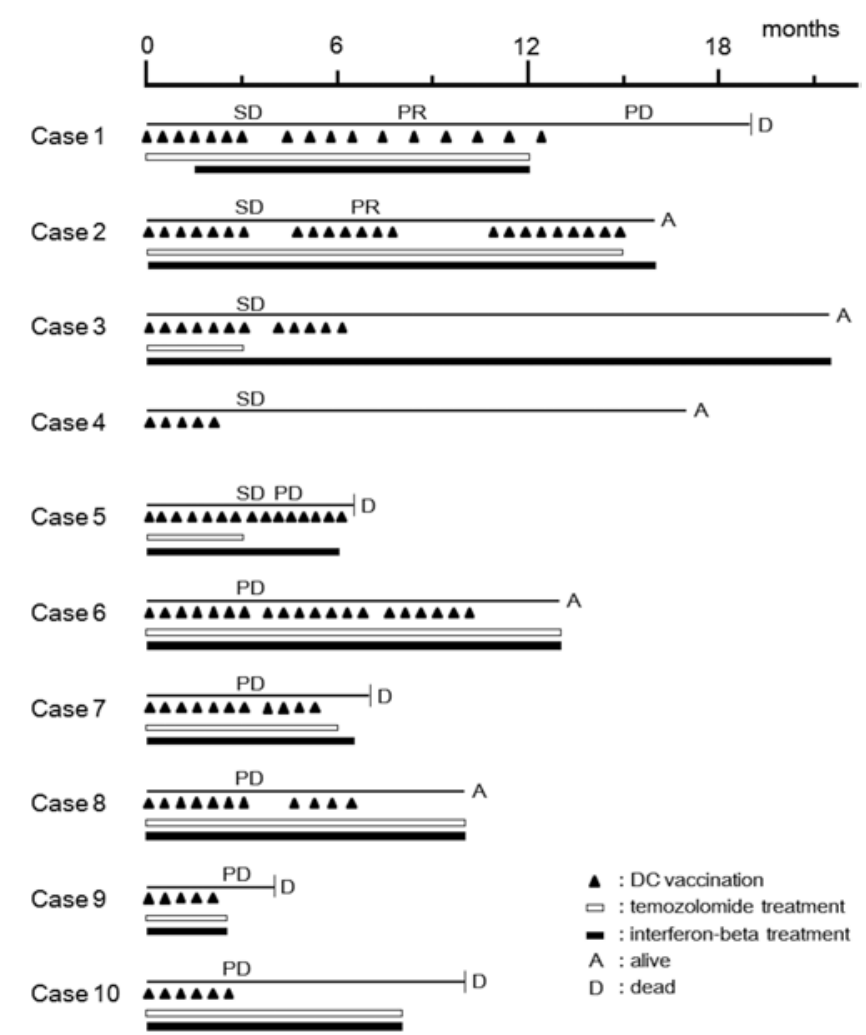

FIG. 2. Response characteristics and applied treatments from the first DC vaccination in each patient. Tumor regression was assessed by using MRI according to the RECIST at the end of the first vaccination session and during the clinical course. The white bars indicate the duration of oral temozolomide treatment $\left(150-200 \mathrm{mg} / \mathrm{m}^{2}\right.$ daily for 5 days every 28 days). The black bars indicate the duration of intravenous interferon$\beta$ treatment (3 million IU/body every 2 weeks).

WT1-specific T cells may have occurred spontaneously in our patients. However, they were distinctly detected and increased after DC vaccination, indicating that our WT1pulsed DC vaccination may have enhanced the cytokine response in our patients. Kaplan-Meier curves showed slow regression. Takahashi et al. ${ }^{43}$ reported prolonged survival of patients with non-small cell lung cancers after treatment with DCs pulsed with the WT1 peptide antigen. A delayed separation of Kaplan-Meier survival curves is observed in almost all randomized immunotherapy trials and may occur months after the start of treatment. ${ }^{14}$

In most clinical trials, autologous DCs are induced to mature and loaded with tumor lysate, tumor cells, or tumor-specific peptides and then infused into the patient. ${ }^{4}$ Yamanaka et al. ${ }^{47}$ administered both autologous mature DCs and autologous immature DCs. In a Phase I/II trial, 22 patients with recurrent malignant gliomas were immunized with $\alpha$-Type 1 polarized DCs $(\alpha \mathrm{DC} 1)$ loaded with synthetic peptides for glioma-associated antigen epitopes and given polyinosinic-polycytidylic acid stabilized with lysine and carboxymethyl cellulose (poly-ICLC). ${ }^{30}$ Glioma-associated antigens include erythropoietin-producing hepatocellular receptor tyrosine kinase Class A2, IL-13 receptor $\alpha 2$, YKL-40, and gp100. Two patients in that trial experienced objective clinical tumor regression (response
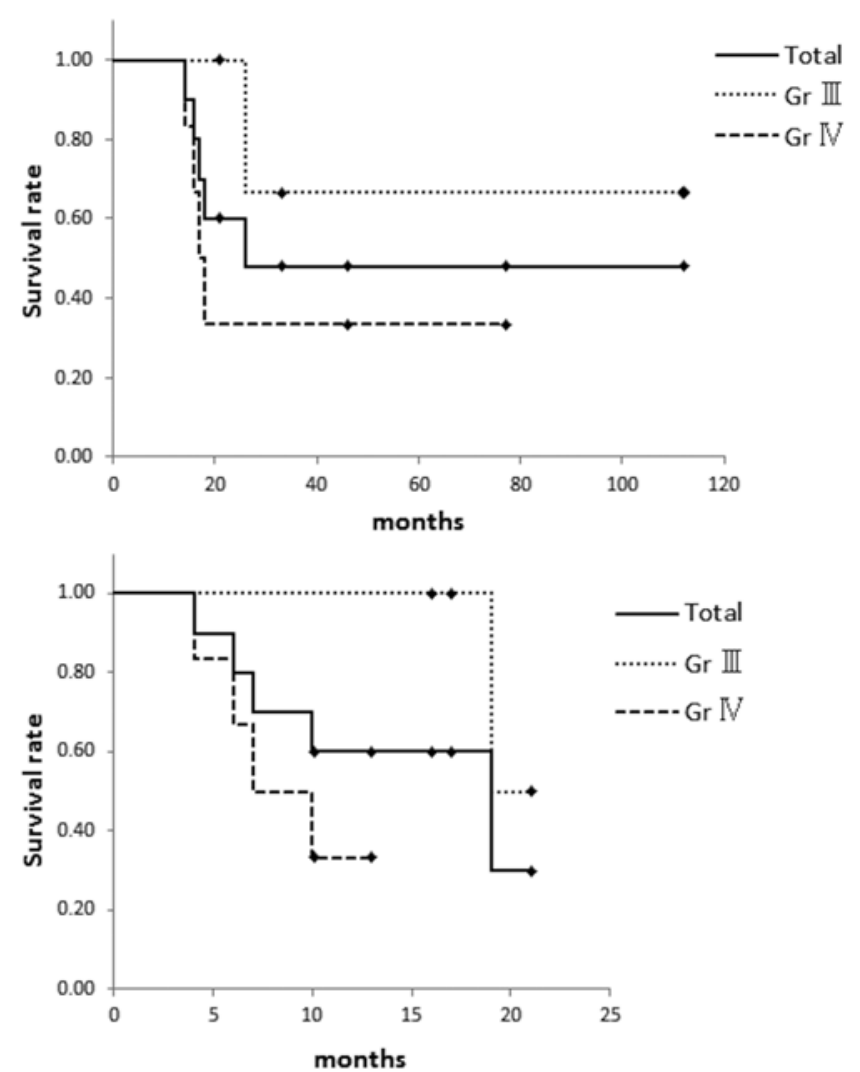

FIG. 3. Upper: Kaplan-Meier curves for overall survival from initial diagnosis of patients with relapsed malignant glioma $(n=10)$ of Grade III $(n=$ 4) or Grade IV (MST 18 months; $n=6$ ). Lower: Kaplan-Meier curves for overall survival from the first vaccination of patients with recurrent malignant glioma $(n=10)$ of Grade III $(n=4)$ or Grade IV (MST 7 months; $n=$ 6). $\mathrm{Gr}=$ Grade.

rate 9\%). The authors reported that the data supported the safety, immunogenicity, and preliminary clinical activity of poly-ICLC-boosted $\alpha$ DC1-based vaccines. Our protocol using mature DCs pulsed with WT1 peptide against malignant glioma has not been reported previously. The safety and efficacy of this protocol in patients with advanced non-small cell lung carcinoma and advanced pancreatic carcinoma have been reported. ${ }^{18,43}$

The most important consideration in DC-based immunotherapy is the most appropriate peptide or antigen with which to pulse the DCs that will optimally boost the tumorspecific CTL response. ${ }^{11}$ Clinical trials of vaccine therapy using a WT1 peptide targeting the WT1 gene product have been performed in patients with various types of carcinomas, including malignant gliomas. ${ }^{28,29} \mathrm{~A}$ Phase I clinical trial was performed in which $3 \mathrm{mg}$ of mutant WT1 peptide was given every week to evaluate its safety. ${ }^{23}$ In 10 patients who received the peptide vaccine, no Grade 3 or 4 toxicities were observed. In addition, Grade 1 and 2 toxicities were acceptable and were considered not to have been caused by the WT1 peptide itself. A PR was observed in 1 patient (with glioblastoma), and SD was seen in 5 patients (4 with glioblastomas and 1 with breast cancer) according to the RECIST. WT1 peptide-based vaccine therapy in patients with WT1/HLA-A*24:02-positive recurrent glioblastomas 
was safe and produced a clinical response. ${ }^{15}$ In this Phase I/II trial, complete response plus PR was achieved in 9.5\% of the patients, and complete response plus PR plus SD was achieved in $57.1 \%$. The results were better than those from previous reports regarding treatment for recurrent glioblastomas. WT1 expression was detected in 70 (95.9\%) of 73 glial tumors examined. ${ }^{12}$ Targeting WT1 may be particularly appropriate, because WT1 is expressed frequently in glioma tissue and is highly immunogenic for adaptive antitumor immune responses. ${ }^{6,29,41}$

The timing of administration is another important factor. The majority of vaccinations were started within 1 week to 3 months after resection. . $^{3,416,32}$ However, previous reports did not describe the time of initiation of vaccination in detail. ${ }^{4}$ In our study, 5 of 6 patients with glioblastoma had PD at the end of the first session of vaccination. Tumor growth in relapsed glioblastomas was so rapid that the performance status progressively deteriorated. With the DC vaccination approach, DCs are extracted from the patient and exposed to antigens expressed by the tumor type to be treated. A couple of months are needed before the initial vaccination can begin because of the need to obtain informed consent and the time needed to manufacture the DC vaccines. Vaccination is recommended to begin as early as possible after tumor recurrence or after resection, especially in patients with glioblastoma., ${ }^{5,47,48}$ Therefore, clinical trials on patients with an initial diagnosis of glioblastoma should be planned.

Some important factors need to be considered regarding DC-based vaccination. In Patient 4, the number of DCs manufactured was extremely low compared with the others. She had received long-term oral steroids for a skin disease before the onset of her brain tumor. Vaccinated patients should be free of corticosteroid administration during all blood collections and vaccinations if possible. Limitations to the use of DC vaccination include the time, expense, and relatively low yield of isolated DCs, as well as the time required for culture and priming of the cells. Standardization of DC vaccine-manufacturing technology and quality analysis, optimization of the selection of artificial antigen peptides, and immunological efficacy analysis will be required for DC-based vaccination therapy to become standard.

Our preliminary study of patients with relapsed malignant glioma has several limitations, such as a small sample size and a group of patients who were heterogeneous with regard to histology, adjuvant therapy, and time of vaccination. However, combined treatment with WT1-pulsed DC-based vaccination and chemotherapy may be feasible, well tolerated, and promising for patients with malignant glioma. Evaluation of the clinical course after vaccination suggested that this vaccination has beneficial effects for some patients. We must stress that the efficacy and safety of DC vaccination should be evaluated using large numbers of patients and prospectively designed studies.

\section{Conclusions}

DC-based immunotherapy targeting WT1 was safe and feasible for the management of advanced malignant gliomas. Based on the results of our pilot study, we will con- tinue developing more advanced clinical trials in patients with malignant gliomas.

\section{Acknowledgments}

We thank Hiromasa Hasegawa, DDS, PhD, Yasuhiro Kobayashi, DDS, PhD, Teruhito Yamashita, PhD, Toshihide Mizoguchi, $\mathrm{PhD}$, and Yuko Nakamichi, PhD (Institute for Oral Science, Matsumoto Dental University, Shiojiri, Japan), for their excellent technical assistance.

\section{References}

1. Adamson C, Kanu OO, Mehta AI, Di C, Lin N, Mattox AK, et al: Glioblastoma multiforme: a review of where we have been and where we are going. Expert Opin Investig Drugs 18:1061-1083, 2009

2. Ardon H, Van Gool S, Lopes IS, Maes W, Sciot R, Wilms G, et al: Integration of autologous dendritic cell-based immunotherapy in the primary treatment for patients with newly diagnosed glioblastoma multiforme: a pilot study. J Neurooncol 99:261-272, 2010

3. Ardon H, Van Gool SW, Verschuere T, Maes W, Fieuws S, Sciot R, et al: Integration of autologous dendritic cell-based immunotherapy in the standard of care treatment for patients with newly diagnosed glioblastoma: results of the HGG-2006 phase I/II trial. Cancer Immunol Immunother 61:20332044, 2012

4. Bregy A, Wong TM, Shah AH, Goldberg JM, Komotar RJ: Active immunotherapy using dendritic cells in the treatment of glioblastoma multiforme. Cancer Treat Rev 39:891-907, 2013

5. Chang CN, Huang YC, Yang DM, Kikuta K, Wei KJ, Kubota T, et al: A phase I/II clinical trial investigating the adverse and therapeutic effects of a postoperative autologous dendritic cell tumor vaccine in patients with malignant glioma. J Clin Neurosci 18:1048-1054, 2011

6. Cheever MA, Allison JP, Ferris AS, Finn OJ, Hastings BM, Hecht TT, et al: The prioritization of cancer antigens: a national cancer institute pilot project for the acceleration of translational research. Clin Cancer Res 15:5323-5337, 2009

7. De Vleeschouwer S, Fieuws S, Rutkowski S, Van Calenbergh F, Van Loon J, Goffin J, et al: Postoperative adjuvant dendritic cell-based immunotherapy in patients with relapsed glioblastoma multiforme. Clin Cancer Res 14:3098-3104, 2008

8. Fadul CE, Fisher JL, Hampton TH, Lallana EC, Li Z, Gui $\mathrm{J}$, et al: Immune response in patients with newly diagnosed glioblastoma multiforme treated with intranodal autologous tumor lysate-dendritic cell vaccination after radiation chemotherapy. J Immunother 34:382-389, 2011

9. Figdor CG, de Vries IJ, Lesterhuis WJ, Melief CJ: Dendritic cell immunotherapy: mapping the way. Nat Med 10:475480, 2004

10. Fujiki F, Oka Y, Tsuboi A, Kawakami M, Kawakatsu M, Nakajima $\mathrm{H}$, et al: Identification and characterization of a WT1 (Wilms Tumor Gene) protein-derived HLA-DRB1*0405restricted 16-mer helper peptide that promotes the induction and activation of WT1-specific cytotoxic T lymphocytes. J Immunother 30:282-293, 2007

11. Gu JH, Li G: Dendritic cell-based immunotherapy for malignant glioma. Neurosci Bull 24:39-44, 2008

12. Hashiba T, Izumoto S, Kagawa N, Suzuki T, Hashimoto N, Maruno M, et al: Expression of WT1 protein and correlation with cellular proliferation in glial tumors. Neurol Med Chir (Tokyo) 47:165-170, 2007

13. Heimberger AB, Sun W, Hussain SF, Dey M, Crutcher L, 
Aldape K, et al: Immunological responses in a patient with glioblastoma multiforme treated with sequential courses of temozolomide and immunotherapy: case study. Neuro Oncol 10:98-103, 2008

14. Hoos A: Evolution of end points for cancer immunotherapy trials. Ann Oncol 23 (Suppl 8): viii47-viii52, 2012

15. Izumoto S, Tsuboi A, Oka Y, Suzuki T, Hashiba T, Kagawa $\mathrm{N}$, et al: Phase II clinical trial of Wilms tumor 1 peptide vaccination for patients with recurrent glioblastoma multiforme. J Neurosurg 108:963-971, 2008

16. Jie X, Hua L, Jiang W, Feng F, Feng G, Hua Z: Clinical application of a dendritic cell vaccine raised against heat-shocked glioblastoma. Cell Biochem Biophys 62:91-99, 2012

17. Keilholz U, Letsch A, Busse A, Asemissen AM, Bauer S, Blau IW, et al: A clinical and immunologic phase 2 trial of Wilms tumor gene product 1 (WT1) peptide vaccination in patients with AML and MDS. Blood 113:6541-6548, 2009

18. Kimura Y, Tsukada J, Tomoda T, Takahashi H, Imai K, Shimamura K, et al: Clinical and immunologic evaluation of dendritic cell-based immunotherapy in combination with gemcitabine and/or S-1 in patients with advanced pancreatic carcinoma. Pancreas 41:195-205, 2012

19. Kobayashi M, Sakabe T, Abe H, Tanii M, Takahashi H, Chiba A, et al: Dendritic cell-based immunotherapy targeting synthesized peptides for advanced biliary tract cancer. J Gastrointest Surg 17:1609-1617, 2013

20. Kuwabara K, Nishishita T, Morishita M, Oyaizu N, Yamashita S, Kanematsu T, et al: Results of a phase I clinical study using dendritic cell vaccinations for thyroid cancer. Thyroid 17:53-58, 2007

21. Li Z, Oka Y, Tsuboi A, Masuda T, Tatsumi N, Kawakami M, et al: WT1(235), a ninemer peptide derived from Wilms' tumor gene product, is a candidate peptide for the vaccination of HLA-A*0201-positive patients with hematopoietic malignancies. Int J Hematol 82:458-459, 2005

22. Melief CJ: Cancer immunotherapy by dendritic cells. Immunity 29:372-383, 2008

23. Morita S, Oka Y, Tsuboi A, Kawakami M, Maruno M, Izumoto S, et al: A phase I/II trial of a WT1 (Wilms' tumor gene) peptide vaccine in patients with solid malignancy: safety assessment based on the phase I data. Jpn J Clin Oncol 36:231-236, 2006

24. Nagayama H, Sato K, Morishita M, Uchimaru K, Oyaizu $\mathrm{N}$, Inazawa $\mathrm{T}$, et al: Results of a phase I clinical study using autologous tumour lysate-pulsed monocyte-derived mature dendritic cell vaccinations for stage IV malignant melanoma patients combined with low dose interleukin-2. Melanoma Res 13:521-530, 2003

25. Nakatsuka S, Oji Y, Horiuchi T, Kanda T, Kitagawa M, Takeuchi T, et al: Immunohistochemical detection of WT1 protein in a variety of cancer cells. Mod Pathol 19:804-814, 2006

26. Nishida S, Hosen N, Shirakata T, Kanato K, Yanagihara M, Nakatsuka S, et al: AML1-ETO rapidly induces acute myeloblastic leukemia in cooperation with the Wilms tumor gene, WT1. Blood 107:3303-3312, 2006

27. Oji Y, Suzuki T, Nakano Y, Maruno M, Nakatsuka S, Jomgeow T, et al: Overexpression of the Wilms' tumor gene W T1 in primary astrocytic tumors. Cancer Sci 95:822-827, 2004

28. Oka Y, Tsuboi A, Oji Y, Kawase I, Sugiyama H: WT1 peptide vaccine for the treatment of cancer. Curr Opin Immunol 20:211-220, 2008

29. Oka Y, Tsuboi A, Taguchi T, Osaki T, Kyo T, Nakajima H, et al: Induction of WT1 (Wilms' tumor gene)-specific cytotoxic T lymphocytes by WT1 peptide vaccine and the resultant cancer regression. Proc Natl Acad Sci U S A 101:1388513890,2004

30. Okada H, Kalinski P, Ueda R, Hoji A, Kohanbash G, Donegan TE, et al: Induction of CD8+ T-cell responses against novel glioma-associated antigen peptides and clinical activity by vaccinations with alpha-type 1 polarized dendritic cells and polyinosinic-polycytidylic acid stabilized by lysine and carboxymethylcellulose in patients with recurrent malignant glioma. J Clin Oncol 29:330-336, 2011

31. Okamoto M, Furuichi S, Nishioka Y, Oshikawa T, Tano T, Ahmed SU, et al: Expression of toll-like receptor 4 on dendritic cells is significant for anticancer effect of dendritic cell-based immunotherapy in combination with an active component of OK-432, a streptococcal preparation. Cancer Res 64:5461-5470, 2004

32. Prins RM, Soto H, Konkankit V, Odesa SK, Eskin A, Yong WH, et al: Gene expression profile correlates with T-cell infiltration and relative survival in glioblastoma patients vaccinated with dendritic cell immunotherapy. Clin Cancer Res 17:1603-1615, 2011

33. Saikali S, Avril T, Collet B, Hamlat A, Bansard JY, Drenou $\mathrm{B}$, et al: Expression of nine tumour antigens in a series of human glioblastoma multiforme: interest of EGFRvIII, IL13Ralpha2, gp100 and TRP-2 for immunotherapy. J Neurooncol 81:139-148, 2007

34. Saito S, Yanagisawa R, Yoshikawa K, Higuchi Y, Koya T, Yoshizawa K, et al: Safety and tolerability of allogeneic dendritic cell vaccination with induction of Wilms tumor 1specific $\mathrm{T}$ cells in a pediatric donor and pediatric patient with relapsed leukemia: a case report and review of the literature. Cytotherapy 17:330-335, 2015

35. Sampson JH, Archer GE, Mitchell DA, Heimberger AB, Bigner DD: Tumor-specific immunotherapy targeting the EGFRvIII mutation in patients with malignant glioma. Semin Immunol 20:267-275, 2008

36. Sampson JH, Heimberger AB, Archer GE, Aldape KD, Friedman AH, Friedman HS, et al: Immunologic escape after prolonged progression-free survival with epidermal growth factor receptor variant III peptide vaccination in patients with newly diagnosed glioblastoma. J Clin Oncol 28:4722-4729, 2010

37. Scheibenbogen C, Letsch A, Thiel E, Schmittel A, Mailaender V, Baerwolf S, et al: CD8 T-cell responses to Wilms tumor gene product WT1 and proteinase 3 in patients with acute myeloid leukemia. Blood 100:2132-2137, 2002

38. Stewart LA: Chemotherapy in adult high-grade glioma: a systematic review and meta-analysis of individual patient data from 12 randomised trials. Lancet 359:1011-1018, 2002

39. Stupp R, Hegi ME, Mason WP, van den Bent MJ, Taphoorn MJ, Janzer RC, et al: Effects of radiotherapy with concomitant and adjuvant temozolomide versus radiotherapy alone on survival in glioblastoma in a randomised phase III study: 5-year analysis of the EORTC-NCIC trial. Lancet Oncol 10:459-466, 2009

40. Stupp R, Mason WP, van den Bent MJ, Weller M, Fisher B, Taphoorn MJ, et al: Radiotherapy plus concomitant and adjuvant temozolomide for glioblastoma. N Engl J Med 352:987-996, 2005

41. Sugiyama H: Cancer immunotherapy targeting Wilms' tumor gene WT1 product. Expert Rev Vaccines 4:503-512, 2005

42. Sugiyama H: Wilms' tumor gene WT1: its oncogenic function and clinical application. Int J Hematol 73:177-187, 2001

43. Takahashi H, Okamoto M, Shimodaira S, Tsujitani S, Nagaya M, Ishidao T, et al: Impact of dendritic cell vaccines pulsed with Wilms' tumour-1 peptide antigen on the survival of patients with advanced non-small cell lung cancers. Eur J Cancer 49:852-859, 2013

44. Vauleon E, Avril T, Collet B, Mosser J, Quillien V: Overview of cellular immunotherapy for patients with glioblastoma. Clin Dev Immunol 2010:1-18, 2010

45. Wheeler CJ, Black KL, Liu G, Mazer M, Zhang XX, Pepkowitz S, et al: Vaccination elicits correlated immune and 
clinical responses in glioblastoma multiforme patients. Cancer Res 68:5955-5964, 2008

46. Yamanaka R: Dendritic-cell- and peptide-based vaccination strategies for glioma. Neurosurg Rev 32:265-273, 2009

47. Yamanaka R, Homma J, Yajima N, Tsuchiya N, Sano M, Kobayashi T, et al: Clinical evaluation of dendritic cell vaccination for patients with recurrent glioma: results of a clinical phase I/II trial. Clin Cancer Res 11:4160-4167, 2005

48. Yu JS, Liu G, Ying H, Yong WH, Black KL, Wheeler CJ: Vaccination with tumor lysate-pulsed dendritic cells elicits antigen-specific, cytotoxic T-cells in patients with malignant glioma. Cancer Res 64:4973-4979, 2004

\section{Author Contributions}

Conception and design: Sakai, Shimodaira, Maejima. Acquisition of data: Sakai, Shimodaira, Maejima, Udagawa, Sano, Higuchi, Koya, Ochiai, Koide, Uehara, Nakamura. Analysis and interpretation of data: Sakai, Shimodaira, Maejima, Sano. Drafting the arti- cle: Sakai, Shimodaira. Critically revising the article: all authors. Reviewed submitted version of manuscript: all authors. Approved the final version of the manuscript on behalf of all authors: Sakai. Administrative/technical/material support: Udagawa, Sano, Higuchi, Koya, Ochiai, Koide, Uehara, Nakamura, Sugiyama, Yonemitsu, Okamoto. Study supervision: Sakai, Shimodaira.

\section{Supplemental Information}

\section{Proceedings}

Portions of this work were presented in partial form as preliminary data at the 2012 Annual Scientific Meeting of the American Association of Neurological Surgeons held in Miami, FL, on April 14-18, 2012.

\section{Correspondence}

Keiichi Sakai, Department of Neurosurgery, Shinshu University School of Medicine, Asahi 3-1-1, Matsumoto, Nagano 390-8621, Japan.email: skeiichi@shinshu-u.ac.jp. 